\title{
Journey of a fish from the vagina to the bladder: a case report
}

\section{Gupta Yogendra B., Anand Kumar I., Ramesh G., Senthil Kumar S., Sudhakar S., Srikala Prasad T.*}

Department of Urology, Chengalpattu Medical College, Tamil Nadu, India

Received: 22 July 2021

Accepted: 13 August 2021

\author{
*Correspondence: \\ Dr. Srikala Prasad T., \\ E-mail: drsrikala@yahoo.co.in
}

Copyright: (c) the author(s), publisher and licensee Medip Academy. This is an open-access article distributed under the terms of the Creative Commons Attribution Non-Commercial License, which permits unrestricted non-commercial use, distribution, and reproduction in any medium, provided the original work is properly cited.

\begin{abstract}
Foreign bodies in the genitourinary tracts are not uncommon and is often a challenge to remove them from the bladder. This case is a peculiar one where there was a migration of a live fish from the vagina to the bladder. A 60- year- old post-menopausal woman while taking bath in a pond felt that there was an entry of fish into the vagina. Her complaints were pain and bleeding per vagina and there was no leakage of urine. At the peripheral health facility, she underwent exploration of the vagina under local anesthesia after placing the foley catheter which was draining blood-stained urine. The patient was then referred to us. There was a laceration at the right anterolateral fornix. Fish could be palpated with the finger. She underwent routine blood tests and CT KUB which revealed a foreign body at the right anterolateral side of the vagina. Cystoscopy showed a laceration of $1 \times 1 \mathrm{~cm}$ noted in the posterior wall of bladder approximately $1 \mathrm{~cm}$ above right ureteric orifice, through which the fish was seen. Through speculum examination, the rent was visible and the remnants of the fish were removed vaginally. Laparotomy and rent repair was done because of the rent in the bladder. Foreign bodies into the bladder are common and invariably seen among children, women who have psychiatric problems. Common foreign bodies seen are broomsticks, toothbrushes, pencils, gauze, sutures, clips, IUCD, etc. Fish as a foreign body is a very rare condition. The unusual entry of a fish into the vagina has eroded and entered the bladder. Prompt investigations like CT scans and cystoscopy can help in the removal of foreign bodies.
\end{abstract}

Keywords: Foreign body, Bladder, Fish, Surgical correction

\section{INTRODUCTION}

Cases of foreign bodies in the genitourinary tract are always of interest. As the occurrence is not uncommon urologists should be familiar with the diagnosis and management of such cases. The bladder is the main receptacle of foreign bodies of the genitourinary tract. A review of world literature on this subject shows that many forms of the foreign body have been removed from the bladder and each one presents a fresh challenge to the urologist. ${ }^{1}$ Introduction of a foreign body into the bladder may be through self-insertion, iatrogenic, traumatic, or migration from adjacent organs with self-insertion being the most common. ${ }^{2}$ Here we report a rare case, where a live fish spontaneously entered into the vagina and passed into the bladder. Our case is unique in that migration of a fish from the vagina to the bladder has not been reported in the literature so far.

\section{CASE REPORT}

A 60 years old multiparous, postmenopausal (10 years back) woman was referred to our medical college hospital at night from a peripheral health facility with chief complaints of severe vaginal pain and bleeding, following an alleged history of spontaneous fish entry into her vagina while taking a bath in a pond near her residence in the evening of the same day. The referring doctor, after placing an 18 Fr Foley catheter had also removed parts of the fish (tail of fish along with scales) per vaginum under local anesthesia. On examination, the patient was found to be mentally sound with no history of any illicit drug or alcohol abuse, or psychiatric illness. The patient denied 
any history of sexual assault or gratification practice. There was no complaint of difficulty in passing urine, straining, dribbling of urine, and urinary leak per vaginum. She had no lower abdominal pain or distention of the abdomen or passage of blood or clot in urine. On examination, her vitals were stable. The abdomen was soft, non-tender, non-distended, and normal bowel sounds were present. On local examination, an 18 Fr Foley catheter was draining mild blood-stained urine. On P/V examination, a laceration of size $2 \times 1 \mathrm{~cm}$ was noted in the right anterolateral fornix at $10-11$ o'clock position, through which the remnants of the fish could be palpated with the finger.

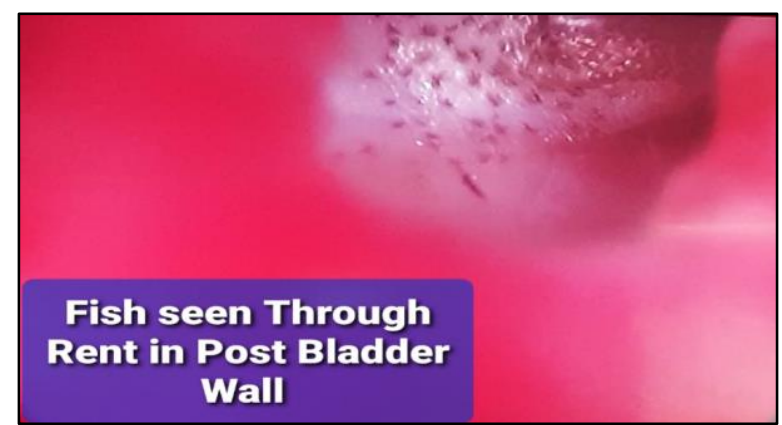

Figure 1: Diagnostic cystoscopy.

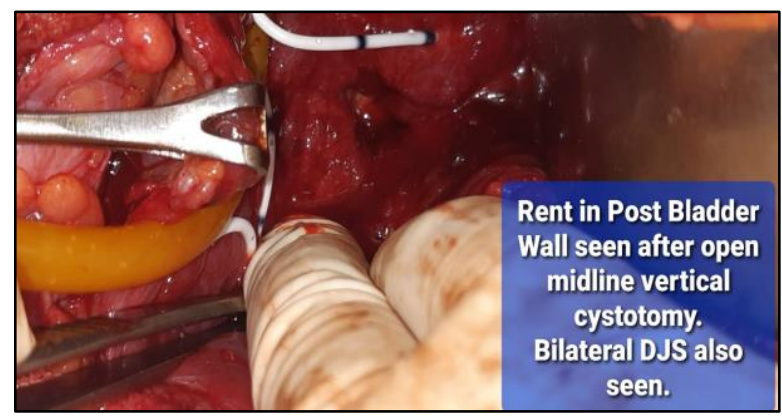

Figure 2: Rent in post bladder wall seen after open midline vertical cystostomy.

Apart from routine blood and urine investigations, noncontrast CT KUB was advised which revealed a hyperdense foreign body, located in the right anterolateral aspect of the vagina (Figure 3). The Foley bulb was identified in the collapsed bladder and without there was no fluid or hematoma in the pelvic cavity. As there were no complaints of a urinary leak or supra pubic pain or tenderness at any point in time, we were not expecting any bladder injury. So patient was planned for diagnostic cystoscopy and exploration under anesthesia.

With 20 Fr sheath and 30-degree telescope, cystoscopy was done and to our surprise a laceration of size approximately $1 \times 1 \mathrm{~cm}$ was noted in the posterior wall of bladder approximately $1 \mathrm{~cm}$ above right ureteric orifice, through which silvery scales of impinged fish was shining (Figure 1).

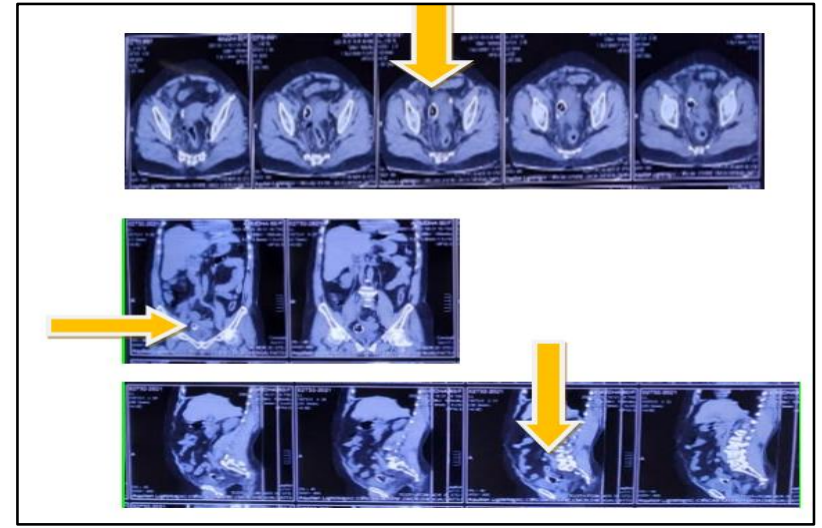

Figure 3: Hyperdense foreign body (Arrow) seen in Axial, Coronal and Sagittal section of CT KUB.

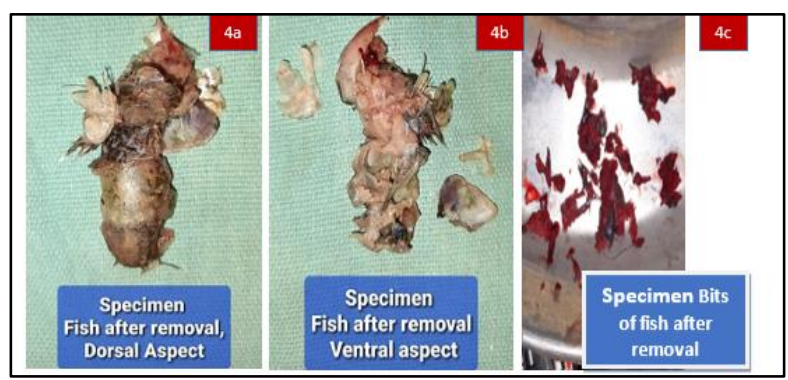

Figure 4: (a) Specimen Fish after removal, dorsal aspect, (b) specimen Fish after removal ventral aspect, (c) specimen bits of fish after removal.

The rest of the cystoscopic findings were normal. With the help of Sims speculum in the vagina, laceration of size $2 \times 1$ $\mathrm{cm}$ was noted in the right anterolateral fornix at $10-11$ o'clock position, through which fish remnants could be palpated. With the help of ovum forceps and finger guidance, the same was removed which was found to be the head and body of fish of size approximately $8 \times 3 \mathrm{~cm}$ (Figure 4a, 4b, 4c). Vaginoscopy was done with a cystoscope and the remaining bits of fish were removed with the help of cystoscopic grasper under vision. Complete removal of fish was confirmed by repeat Cystoscopy, which did not show any fish remnants, and only the laceration in the posterior wall of the bladder was seen.

The abdomen was opened with a Pfannenstiel incision and the bladder was identified. The pelvic cavity was explored and no other foreign body or part of fish was found. The bladder was opened by a vertical midline incision, and confirming the findings of cystoscopy, a laceration of size approximately $1 \times 1 \mathrm{~cm}$ was seen in the posterior wall of the bladder approximately $1 \mathrm{~cm}$ above the right ureteric orifice. Bladder laceration was repaired with continuous 2-0 delayed absorbable running suture after prophylactic bilateral DJ Stenting (Figure 2). Vertical midline cystotomy was closed after placing 20 Fr Foley catheter as Supra Pubic Catheter (SPC). The abdomen was closed with a pelvic drain. Vaginal wall laceration was left to heal by secondary intention. The postoperative period was 
uneventful. Pelvic drain, SPC, PUC, and B/L DJS removal was done on POD 3, 2 weeks, 3 weeks, and 8 weeks respectively. The patient is doing well at 2 months followup.

\section{DISCUSSION}

The urinary bladder is the most common site for lodgement of foreign bodies as they are typically propelled into the bladder through the urethra, as most of the foreign bodies are self-introduced by the patient itself typically for sexual eroticism, curiousness (particularly in children), as a consequence of disinhibited or disturbed behavior (example- psychiatric illness) or under the influence of drugs or alcohol.

Myriads of foreign bodies noted within the bladder encompass everything available within the social surroundings of the self-inserter, from broom straws and perfume bottle to toothbrush and pencil., ${ }^{1,3-6}$

Some foreign bodies found in the bladder are iatrogenic such as sutures, clips, and gauze. Some foreign bodies will be introduced into the bladder by migration from adjacent structures, like gauze, Intra uterine contraceptive device (IUCD) and pessary.

Regarding fish as a foreign body in the urinary bladder, there is a case report where a young boy was holding a fish in his hand while he was passing urine and the fish slipped from his hand and entered his urethra, and reached the bladder. $^{7}$

In the majority of cases clinical presentation varies from being asymptomatic to swelling of the external genitalia, dysuria, poor urinary stream or retention, bloody or purulent urethral discharge, and ascending urinary tract infection and depends on characteristics of the object, location, method of introduction, duration as well as associated injury. ${ }^{8,9}$ In our case, the chief complaint was severe vaginal pain and bleeding. Diagnosis, as in our case, is typically made from careful clinical history (history of foreign body insertion) and examination along with radiological studies (X-Ray KUB, CT KUB).

The treatment of the foreign body in the urinary bladder ought to be aimed towards complete removal, following rules of standard surgical practice. Extraction should be tailored in line with the character of the foreign body and should cause minimal trauma to the bladder and urethra. Removal can be performed either using a cystoscope or by open surgical exploration. The rent in the anterolateral aspect of the vagina helped us retrieve the remnants of the fish through the vagina. Complete removal should also be confirmed by endoscopy at the end of the extraction procedure..$^{8-10}$

\section{CONCLUSION}

The world literature abounds with reports of intravesical foreign bodies. Our case is a peculiar one as there is no case report where an intravesical foreign body is a fish, introduced into the bladder through the vagina by spontaneous migration of a living fish.

Funding: No funding sources

Conflict of interest: None declared

Ethical approval: Not required

\section{REFERENCES}

1. Van Ophoven A, De Kernion JB. Clinical management of foreign bodies of the genitourinary tract. The Journal of urology. 2000;164(2):274-87.

2. Odoemene CA, Onuh CA. Foreign bodies in the urinary bladder-case series. Journal of the West African College of Surgeons. 2017;7(3):124.

3. Van Ophoven A, De Kernion JB. Clinical management of foreign bodies of the genitourinary tract. The Journal of urology. 2000;164 (2):274-87.

4. Duncan IG. Perfume bottle in man's bladder: case report. Memphis Med J. 1927;4:118.

5. Granados EA, Riley G, Rios GJ, Salvador J, Vicente J. Self-introduction of urethra vesical foreign bodies. European urology. 1991;19(3):259-61.

6. Bantis A, Sountoulides P, Kalaitzis C, Giannakopoulos S, Agelonidou E, Foutzitzi S et al. Perforation of the urinary bladder caused by transurethral insertion of a pencil for the purpose of masturbation in a 29 -year-old female. Case reports in medicine. 2010;2010.

7. Balci U, Horsanali MO, Kartalmis M, Girgin C, Dinçel Ç. An unusual foreign body in the urinary bladder: beading awl/Mesanede nadir bir yabanci cisim: Tig. Turkish Journal of Urology. 2011;37(3):275.

8. Eckford SD, Persad RA, Brewster SF, Gingell JC. Intravesical foreign bodies: five-year review. British journal of urology. 1992;69(1):41-5.

9. Bansal A, Yadav P, Kumar M, Sankhwar S, Purkait B, Jhanwar A, Singh S. Foreign bodies in the urinary bladder and their management: a single-centre experience from North India. International neurourology journal. 2016;20(3):260.

10. Rafique M. Intravesical foreign bodies review and current management strategies.

Cite this article as: B GY, I A, G R, Kumar S SK, S $\mathrm{S}$, T SP. Journey of a fish from the vagina to the bladder: a case report. Int J Reprod Contracept Obstet Gynecol 2021;10:3617-9. 